\title{
RPA homologs and ssDNA processing during meiotic recombination
}

\author{
Jonathan Ribeiro ${ }^{1,2,3,4}$ • Emilie Abby ${ }^{1,2,3,4}$ - Gabriel Livera ${ }^{1,2,3,4}$. \\ Emmanuelle Martini ${ }^{1,2,3,4}$
}

Received: 10 April 2015 /Revised: 12 September 2015 / Accepted: 14 October 2015 / Published online: 31 October 2015

(C) The Author(s) 2015. This article is published with open access at Springerlink.com

\begin{abstract}
Meiotic homologous recombination is a specialized process that involves homologous chromosome pairing and strand exchange to guarantee proper chromosome segregation and genetic diversity. The formation and repair of DNA double-strand breaks (DSBs) during meiotic recombination differs from those during mitotic recombination in that the homologous chromosome rather than the sister chromatid is the preferred repair template. The processing of singlestranded DNA (ssDNA) formed on intermediate recombination structures is central to driving the specific outcomes of DSB repair during meiosis. Replication protein A (RPA) is the main ssDNA-binding protein complex involved in DNA metabolism. However, the existence of RPA orthologs in plants and the recent discovery of meiosis specific with OB domains (MEIOB), a widely conserved meiosis-specific RPA1 paralog, strongly suggest that multiple RPA complexes evolved and
\end{abstract}

This article is part of a Special Issue on "Recent advances in meiotic chromosome structure, recombination and segregation" edited by Marco Barchi, Paula Cohen and Scott Keeney

Electronic supplementary material The online version of this article (doi:10.1007/s00412-015-0552-7) contains supplementary material, which is available to authorized users.

Emmanuelle Martini

emmanuelle.martini@cea.fr

1 Laboratory of Development of the Gonads, Unit of Stem Cells and Radiation, University of Paris Diderot, Sorbonne Paris Cité, UMR 967, F-92265 Fontenay-aux-Roses, France

2 CEA, DSV, iRCM, SCSR, LDG, F-92265 Fontenay-aux-Roses, France

3 INSERM, Unité 967, F-92265 Fontenay-aux-Roses, France

4 Université Paris-Saclay, UMR-967, F-92265 Fontenay-aux-Roses, France specialized to subdivide their roles during DNA metabolism. Here we review ssDNA formation and maturation during mitotic and meiotic recombination underlying the meiotic specific features. We describe and discuss the existence and properties of MEIOB and multiple RPA subunits in plants and highlight how they can provide meiosis-specific fates to ssDNA processing during homologous recombination. Understanding the functions of these RPA homologs and how they interact with the canonical RPA subunits is of major interest in the fields of meiosis and DNA repair.

Keywords Meiosis $\cdot$ Recombination $\cdot$ ssDNA $\cdot$ RPA . MEIOB · SPATA22

Meiosis is the central process of sexual reproduction. During this specialized cell division program, the genome of diploid germ cells is halved to produce haploid gametes. This process requires the pairing and formation of a physical link (chiasma) between homologous chromosomes (homologs). These events are established during meiotic prophase I, which is subdivided into four stages (for review see Page and Hawley 2003). Prophase begins with the formation of programmed DNA double-strand breaks (DSBs) during the leptotene stage. During the zygotene and pachytene stages, these DSBs are progressively repaired by homologous recombination (HR), a process that promotes and completes homolog pairing (Weiner and Kleckner 1994; Kauppi et al. 2013). Chiasmata are the product of crossovers (COs) formed during the pachytene stage. Only a subset of DSBs are repaired with a reciprocal exchange of chromosome arms to form a $\mathrm{CO}$, whereas the remaining DSBs are repaired without reciprocal exchange to form noncrossovers (NCOs). COs are tightly regulated as shown by their controlled number and non-random distribution along chromosomes (Anderson et al. 1999; Martini et al. 
2006; Cole et al. 2012). It is now evident that numerous factors are necessary to control the proper progression of meiotic HR and drive the maturation of intermediates to specific outcomes (for review seeYouds and Boulton 2011). First, meiotic DSBs are programmed and formed through the action of several factors including the conserved topoisomerase-like transesterase SPO11, which bears the catalytic activity. DSB initiation was recently shown to be under feedback control by Tel1/ATM kinase (Carballo et al. 2013; Lange et al. 2011; Garcia et al. 2015). During a later step, the conversion of pre-crossover intermediates into COs has been demonstrated to depend on the coordination of a number of proteins (De Muyt et al. 2014; Holloway et al. 2014; Qiao et al. 2014).

Single-stranded DNA (ssDNA) is generated at multiple steps during HR (see Fig. 1) and requires specific factors for its formation, signal transduction, protection, and disappearance. Among these factors, Replication Protein A (RPA) is the principal ssDNA-binding complex and is essential for mitotic growth and meiotic progression (Soustelle et al. 2002). However, recent studies have highlighted the involvement of additional RPA homologs specifically during meiosis, notably meiosis specific with OB domains (MEIOB) a new meiosis-specific paralog of the largest subunit of RPA (Souquet et al. 2013; Luo et al. 2013). In this review we discuss the potential specific roles of RPA and its homologs to better understand the regulation of ssDNA-containing meiotic recombination intermediates.

\section{Roles of ssDNA during homologous recombination}

\section{During the mitotic cell cycle}

ssDNA formation is a common feature of DNA metabolism. It is generated during DNA replication, repair, and recombination (Mehta and Haber 2014). During HR, ssDNA is formed on different intermediates and bound by dedicated proteins that ensure its protection and signaling and the subsequent steps of DNA processing. HR begins with the production of 3 '-ssDNA tails to invade duplex DNA during the homology search. Subsequently, D-loop formation and migration generate additional ssDNA. Shortly after formation, DSBs are recognized by the MRN complex (MRE11, RAD50, and NBS1) to initiate 5'-> 3'- resection (Ivanov et al. 1994; Tsubouchi and Ogawa 1998). To protect ssDNA from degradation and remove secondary structures that would prevent the loading of recombinase proteins, the RPA complex binds the resected DNA (Alani et al. 1992; Sugiyama et al. 1997; Wang and Haber 2004). RPA is subsequently replaced by the RAD51 recombinase to form a presynaptic nucleofilament in a BRCA2- and Rad52-dependent manner in mammals and yeast, respectively, with the help of RAD51 paralogs (Sung 1997; Gasior et al. 2001; Jensen et al. 2010; Suwaki et al. 2011; Gaines et al. 2015). In eukaryotic cells, it is assumed that RPA loading precedes RAD51 loading. However, RAD51 can be loaded on ssDNA in the absence of RPA if the ssDNA is free of secondary structures (Heyer and Kolodner 1989; Sung 1994; Sugiyama et al. 1997; Gaines et al. 2015). The large subunit of RPA interacts with RAD51 through its N-terminal region and co-localizes to sites of DNA damage; however, it remains unclear whether RPA and RAD51 coexist in the nucleofilament (Golub et al. 1998; Tarsounas et al. 2003; Haber 2014).

Owing to its DNA strand-pairing activity, the nucleofilament invades a homologous DNA duplex to initiate DNA synthesis from the invading $3^{\prime}-\mathrm{OH}$ end using the donor sequence as the template. Genetic evidences have revealed that the mismatch repair machinery (MMR) inhibits recombination between moderately divergent regions (Evans and Alani 2000). Studies with mismatches containing substrates incubated in human cell extracts have shown that RPA is recruited at mismatch sites prior to MMR-specific members (Guo et al. 2006). Moreover, the in vitro reconstitution of MMR reaction with purified hMMR proteins has provided evidence that RPA forms part of the complex that initiates mismatch-provoked excision, suggesting that RPA plays a role in the early steps of the MMR. It remains unclear whether RPA collaborates with the MMR machinery in mismatch tolerance during the search for homologous sequences that occurs during HR. Furthermore, whether RPA ensures this role on the nucleofilament or after loading onto the D-loop formed during strand exchange is an open question. Evidence for the presence of RPA on the D-loop has been provided by several observations (Wang and Haber 2004). Biochemical assays showed that RPA strongly enhances strand exchange by stabilizing RAD51-dependent pairing after strand invasion and stimulates DNA synthesis through its interaction with DNA polymerases alpha and delta after invasion (Eggler et al. 2002; Sneeden et al. 2013). RPA-dependent strand invasion stabilization is achieved by preventing reversal of the strand exchange reaction. Chromatin immunoprecipitation of RPA performed in Saccharomyces cerevisiae revealed that RPA is retrieved on both the donor and the recipient sequences with time differences confirming the presence of RPA first on the broken strand and then on the D-loop in vivo (Wang and Haber 2004). Moreover, in vitro studies have indicated that RPA favors strand annealing directed by yeast or human RAD52 (Sugiyama et al. 1998; Jensen et al. 2010). Finally, the importance of RPA during second-end capture has been demonstrated by biochemical assays performed with the $S$. cerevisiae rfal-t11 mutant. This mutant supports the RAD52-dependent loading of RAD51 and strand invasion but exhibited failure in second-end capture (Sugiyama et al. 2006).

\section{During prophase I of meiosis}

Even though meiotic and mitotic HR share common features and factors, several facets of HR differ. DSBs are programmed 


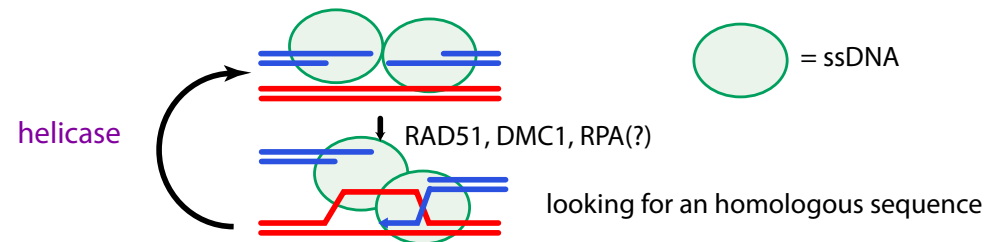

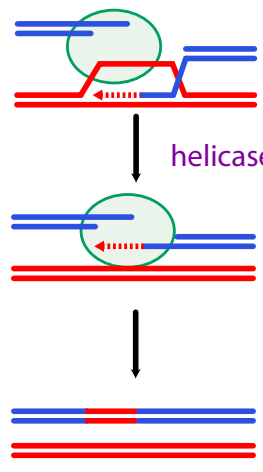

Noncrossover (NCO)
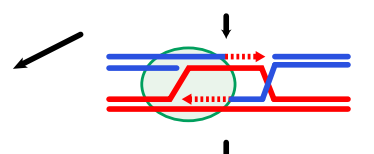

stabilization

$\downarrow$

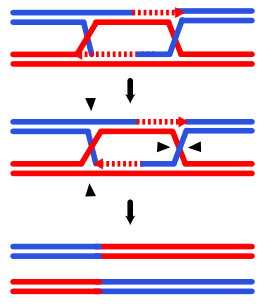

Crossover (CO)

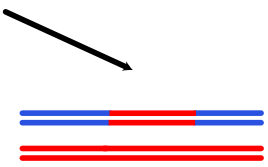

Noncrossover (NCO)

Fig. 1 Meiotic recombination and ssDNA. Meiotic double strand breaks are repaired as crossovers (CO) or non-crossovers (NCOs) through different intermediates. After DSBs formation, resection is initiated to form 3 '-ssDNA tails. The 3'-tail is then coated by the recombinases to invade the homologous sequence on the homolog. A ssDNA-binding protein such as RPA must help to stabilize and protect ssDNA before

during meiosis and involve the presence of specific factors at DSB sites, including before DSB formation. Our knowledge regarding the proteins required for meiotic DSB formation comes mostly from studies performed in S. cerevisiae, which has at least nine proteins that are essential to this process, whereas only three have so far been identified in mice. In $S$. cerevisiae, Mer2 is loaded onto chromatin to coordinate DNA replication and DSB formation in a phosphorylationdependent manner (Henderson et al. 2006; Murakami and Keeney 2014). The remaining proteins are subsequently loaded to induce DSB formation through the catalytic activity of Spo11. Interestingly, the MRX complex (Mre11, Rad50, and Xrs2) which is involved in ssDNA formation to produce an ssDNA $3^{\prime}$ end during mitotic HR, is essential for DSB formation in S. cerevisiae and Caenorhabditis elegans (Borde 2007). This observation indicates that a complex loaded on broken ends during mitotic HR to initiate ssDNA processing is present before DSB formation during meiotic HR. Consequently, the chromatin context in which ssDNA processing occurs differs between both types of HR. Interestingly, although RPA foci are detected on unsynapsed chromosomes suggesting the presence of RPA on ssDNA before strand invasion, the precocious formation of numerous and bright RAD51 foci at the time the first RPA foci are detected may bring into question whether RPA binding on ssDNA precedes the loading of recombinases during meiotic HR in mammals as observed during mitotic HR (Plug et al. 1998; Moens et al. 2002; Oliver-Bonet et al. 2007 and personal observation E.A., the formation of the presynaptic filament. Proper strand invasion is stabilized to initiate DNA synthesis and then either further stabilized or destabilized to be repaired by synthesis-dependent strand annealing (SDSA). The stabilized intermediates (double Holliday junctions) can either form a NCO outcome or be resolved by nuclease activity to form a CO or a NCO outcome. Green circles show ssDNA

EM). Of note, RPA foci have been observed on chromosome spreads performed in a rad50S mutant of $S$. cerevisiae. This mutant is unable to process DNA after DSB formation and is not expected to produce ssDNA suggesting that normal resection is not required to recruit RPA to DSB sites (Gasior et al. 1998). This finding could be explained through the direct interaction described between MRE11 and RPA or by nonspecific DNA degradation (Gasior et al. 1998).

Unlike mitotic recombination, meiotic recombination exhibits preference to use as the repair template a DNA sequence present on the homologous chromosome rather than a DNA sequence on the sister chromatid and favors the formation of COs (Schwacha and Kleckner 1994; Cole et al. 2014). In addition to RAD51, most organisms possess the meiosisspecific DMC1 recombinase, which is essential together with RAD51 for proper strand invasion (Bishop et al. 1992; Yoshida et al. 1998). Studies performed in S. cerevisiae and Arabidopsis thaliana suggest that during meiotic HR, RAD51 would preferentially play an accessory role to allow DMC1 recombinase activity (Cloud et al. 2012; Da Ines et al. 2013). These observations suggest that RAD51 and DMC1 can load together on both broken ends after DSB formation. However, the distances observed between RAD51 and DMC1 foci by immunolocalization in A. thaliana support the hypothesis that distinct filaments would be formed on each end (Kurzbauer et al. 2012). Although a strong effort has been made to decipher the formation, maintenance, and dynamics of the RAD51/DMC1 nucleofilament, further investigations remain 
necessary (Brown and Bishop 2014). Studies performed in plants and mammals indicate that BRCA2 interacts with $\mathrm{DMC} 1$ and RAD51, suggesting that BRCA2 promotes the formation of the meiotic nucleofilament containing RAD51 and DMC1 (Siaud et al. 2004; Thorslund and West 2007; Seeliger et al. 2012). Genetic evidence has confirmed the importance of BRCA2 to mediate the proper formation of the meiotic presynaptic nucleofilament (Sharan et al. 2004). In addition to stimulating nucleofilament formation, yeast RAD52 (yRad52) is required for the post-invasion steps of meiotic HR (Lao et al. 2008). Indeed, the annealing of the second broken end during synthesis-dependent strand annealing (SDSA) and second-end capture to complete the formation of double Holliday junctions (dHJs) are dependent on yRad52 in S. cerevisiae and BRCA-2 in C. elegans (Sugiyama et al. 2006; Petalcorin et al. 2006). However, in vitro assays have demonstrated that hBRCA2 does not anneal RPA-coated ssDNA, whereas hRAD52 does, suggesting that two proteins are needed in mammals compared with one in yeast and C. elegans (Jensen et al. 2010; Petalcorin et al. 2006). Nonetheless, in contrast to what has been described in yeast, knockouts of the Rad52 gene in mammals show few phenotypes with no obvious defect in response to DNA damaging agents and no meiotic defects (Rijkers et al. 1998).

The presence of recombinases on the presynaptic filament is essential for the displacement and invasion of the homologous sequence. However, the nature, organization, and dynamics of the nucleofilament formed on the second broken end remain to be determined in vivo. Given the specific outcomes of this end (i.e., strand annealing or second-end capture) it is unclear whether the recombinases and/or RPA are loaded on that end. Immunostaining of RPA performed on chromosome spreads in numerous species (mammals and plants) have shown that RPA foci are detected up to the pachytene stage, specifically until the formation of MLH1 COassociated foci (Plug et al. 1998). This finding suggests that RPA is present on joint molecules after their stabilization with the MutS homologs, MSH4-MSH5. Interestingly, the phosphoregulation of RPA appears to play a role in the control of CO formation. In S. cerevisiae, the phosphorylation of the RPA2 subunit is controlled by the yeast ATR ortholog, Mec1. A phosphomimetic form of RPA2 induces changes in genetic distances and $\mathrm{CO}$ interference, suggesting a role for RPA2 phosphorylation in CO regulation (Bartrand et al. 2006).

For decades, RPA has been considered a strong unalterable complex that is well conserved among species. However, the identification in plants of variants of the three subunits of RPA that can form different complexes with specific activities strongly underlines the potential regulatory role that such complexes can play (Shultz et al. 2007). The existence of alternative RPA complexes in mammalian cells has been suggested by the discovery of RPA4, which bears $50 \%$ identity with RPA2, in a cDNA library derived from HeLa cells
(Keshav et al. 1995). More recently, it has been proposed that RPA4 is more likely to be involved in the maintenance of genomic integrity than in its replication (Haring et al. 2010). Moreover, recent studies identified a new meiosis-specific gene in metazoans, MEIOB, a paralog of RPA1 that interacts with RPA2 (Souquet et al. 2013; Luo et al. 2013). MEIOB is essential for proper meiotic recombination in mice and interacts with ssDNA. In-depth characterization of these multiple complexes should allow a better understanding of the mechanisms regulating meiotic recombination and the maintenance of genome integrity.

\section{RPA the major ssDNA-binding protein}

\section{Canonical RPA complex}

The ssDNA-binding replication protein A (RPA) is an evolutionarily conserved heterotrimeric complex involved in DNA replication, repair, and recombination which is essential for cell survival (for a review see Wold 1997). RPA was originally identified through its essential role for SV40 DNA replication in vitro (Wold and Kelly 1988).

The RPA complex is composed of three subunits: RPA1, RPA2, and RPA3. X-ray diffraction studies of the crystallized human complex revealed that the three subunits form together a trimer that is stabilized through the trimerization core produced by the interaction of helices from each subunit (Bochkareva et al. 2002). Among the three subunits, RPA1 possesses the highest affinity for ssDNA through its three oligonucleotide/oligosaccharide-binding folds (OB-fold), denoted DBD-A, DBD-B and DBD-C (Wold 1997). RPA2 and RPA3 also possess OB-fold domains, named DBD-D and DBD-E, which are capable of interacting with ssDNA (Philipova et al. 1996; Salas et al. 2009) (see Fig. 2). RPA binds to ssDNA in two conformational states that differ in the length and affinity of the bound DNA. A study of the structure of a crystallized RPA-ssDNA complex from the fungus Ustilago maydis demonstrated that the two conformational states of RPA-ssDNA binding provides opposing affinities for DNA and proteins (Fan and Pavletich 2012). The DBD-C of RPA1 is longer than the two other OB-folds and possesses an insertion of approximately 30 amino acids with a conserved zinc ion-binding domain of type C-4X-C13X-C-2X-C (Heyer et al. 1990; Erdile et al. 1991). This domain is dispensable for RPA1 ssDNA-binding activity but influences the overall ssDNA-binding affinity of the RPA complex (Kim et al. 1996; Dong et al. 1999; Walther et al. 1999). However, the functional role of this domain remains poorly understood. Kim et al. and Walther et al. reported that mutating this domain strongly impaired SV40-dependent DNA replication, whereas Dong et al. observed only a slight delay. 
A

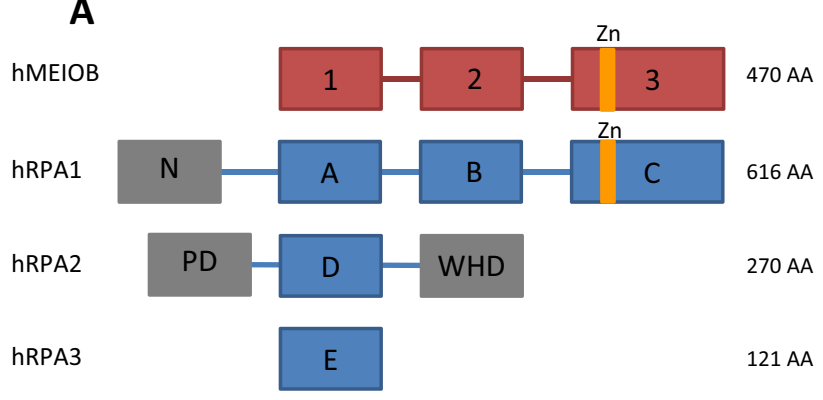

B

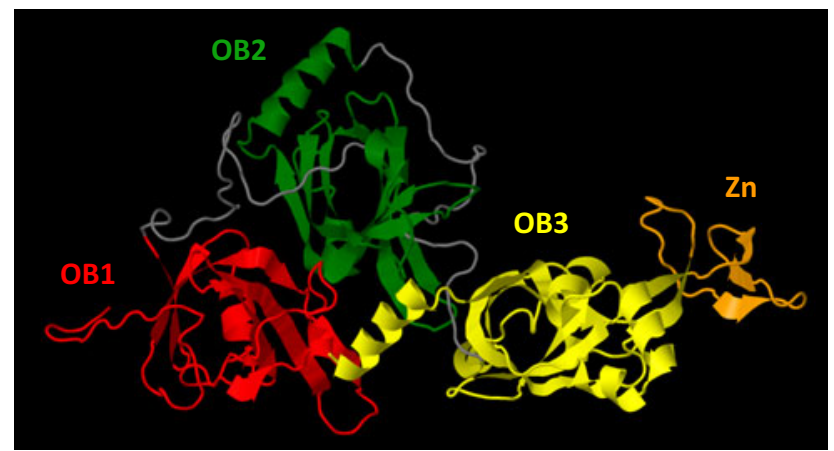

Fig. 2 Domain structures of RPA subunits and MEIOB. a Schematic representation of MEIOB and RPA subunits protein domains. The folded domains and unfolded linkers are presented as boxes and lines respectively. The red and blue boxes represent MEIOB and RPA subunit OB- folds, respectively. The orange domains illustrate zinc ionbinding domains. The grey boxes represent domains that are not involved in ssDNA-binding activity, such as the N-terminal domain of RPA1 and the phosphorylation domain (PD) and the winged-helix domain (WHD) of RPA2. b Stereo ribbon presentation of predicted MEIOB structure model obtained from the RaptorX server (Kallberg et al. 2012) and visualized with Jmol (www.jmol.org). OB-folds 1, 2, and 3 and zinc ion-binding domain are represented in red, green, yellow, and orange, respectively. Unfolded linkers are represented in grey

\section{Existence and properties of multiple RPA complexes}

Genome duplications occurred periodically during eukaryotic evolution such that some organisms possess several homologs of RPA subunits that may have meiosisspecific functions. Such multiple RPAs have been described in the plants A. thaliana and Oryza sativa and in the protists Toxoplasma gondii, Plasmodium falciparum, and Cryptosporidium parvum (Rider et al. 2005; Gopalakrishnan and Kumar 2013).

The flowering plant $A$. thaliana contains five homologs of RPA1, two homologs of RPA2, and two homologs of RPA3. The rice $O$. sativa possesses three homologs of RPA1 and RPA2 and only one homolog of RPA3. Interestingly, despite the multiple possible combinations, only three complexes have been identified in rice and four in A. thaliana, respectively (Ishibashi et al. 2006; Eschbach and Kobbe 2014). These observations suggest the existence of specific interactions between subunits and/or specialized expression that restricts the possibilities of interactions.
Genetic studies performed with mutants of AtRPA1 homologs led to the classification of these homologs into two groups. AtRPA1a, AtRPA1c, and AtRPAle are hypothesized to be involved in the response to DNA-damaging agents and consequently in DNA repair, whereas AtRPA1b and AtRPA1d are proposed to be necessary for normal DNA replication during developmental growth (Aklilu et al. 2014). No meiotic defect could be observed in Atrpalb,c,d,e mutants (Aklilu et al. 2014). A careful genetic study of AtRPAla during meiosis revealed its role in meiotic HR. Although the absence of fragmented DNA suggests that DSBs are repaired in this mutant, immunostaining performed on meiotic chromosome spreads showed a reduction in the MLH1 foci number, strongly suggesting a diminution of interfering COs (class I). Metaphase I analysis confirmed a reduction in the chiasma number. The observed phenotype suggested that meiosis progressed normally until class I CO formation. Therefore, the authors proposed that AtRPAla could be involved in the second-end capture of the broken end at the recombination intermediates designated to form class I COs controlled by MLH1/MLH3 (Osman et al. 2009). Furthermore, mutation of both AtRPAIc and AtRPAIa was found to induce complete sterility and marked chromosome fragmentation during meiosis. The findings that the Atrpala/Atrpalc double mutant is sterile and that the Atrpalc mutant does not show fertility problems suggest that AtRPA1c could play an early role during meiotic recombination that could be overcome by AtRPAla in the absence of AtRPAlc.

Less genetic data are available regarding the functions of RPA1 homologs in rice. To date, only the function of OsRPA la has been studied. The osrpala mutant showed normal vegetative growth but an increased sensitivity to DNA damage induced by genotoxic agents, including UV irradiation. osrpala mutants are sterile and show chromosomal fragmentation despite normal chromosome pairing and synapsis during meiosis (Chang et al. 2009). These data suggest that OsRPA1a is dispensable for replication but required for somatic and meiotic DNA repair.

Not much is known about the biochemical properties of the RPA complexes in rice, however, the DNA binding properties of AtRPA complexes were recently published (Eschbach and Kobbe 2014). The authors concluded that complexes containing AtRPA1a show higher affinity for unmodified ssDNA than ssDNA with modifications, such as abasic sites, and stronger dsDNA-destabilizing activity than AtRPA1bcontaining complexes. These results were unexpected in light of genetic data suggesting that AtRPA1b is specifically involved in DNA replication because the Atrpala mutant did not show growth defects in the absence of DNA-damaging agents. Such results may be explained by the absence of RPA-interacting partners or post-translational modifications in the in vitro assay. In addition, the study revealed that the identity of the RPA3 homolog may significantly influence the 
DNA-binding properties of the complexes. This result was unexpected given the weak DNA-binding affinity of RPA3, a subunit that was proposed to mediate protein-protein interaction (Bochkarev et al. 1999).

Collectively, these genetic and biochemical studies suggest that multiple RPA complexes have evolved and become specialized to subdivide their roles during the various stages of DNA metabolism.

\section{MEIOB: a meiosis-specific RPA1 homolog}

\section{MEIOB and evolution}

For several decades, RPA4, which belongs to the RPA2 family, was the only RPA paralog to have been found in mammals. However, recent works identified Meiob as a new meiosis-specific paralog of Rpal in metazoans (Souquet et al. 2013; Luo et al. 2013). Meiob orthologs have been retrieved in the genomes of almost all metazoans, with the exception of Nematoda and a non-meiotic specific ortholog of MEIOB, named Hold'em (HDM), has been described in Drosophila (Joyce et al. 2009). Interestingly, an ortholog has also been found in the single-celled organism Capsaspora owczarzaki (Souquet et al. 2013). This finding strongly suggests that a duplication event that occurred before the emergence of metazoans and multicellularity enabled Meiob to evolve from an ancestral Rpa1 gene (approximately 600 million years ago). However, we recently identified sequences similar to metazoan Meiob in several fungal genomes such as the zygomycotan fungi Mortierella verticillata, and in genomes of ascomycetes, such as Neurospora crassa, suggesting that the evolution of Meiob may be earlier than first thought (22.3\% identity between human MEIOB and MEIOB-like of $M$. verticillata, see Fig. 3, unpublished data). Deciphering the function of these Meiob-like proteins will help us to better understand the role of MEIOB. To date, we have not identified a Meiob ortholog in amoebozoan genomes. The fact that MEIOB has been retrieved from metazoans, fungi, and Capsasporidae (C. owczarzaki) suggests that the appearance of MEIOB occurred during the evolution of the Opisthokonta monophyletic group. Surprisingly, putative Meiob orthologs have not been identified in the $S$. cerevisiae or Schizosaccharomyces pombe genomes. This could be explained by the loss of an ancestral Meiob gene during the evolution of these species - a situation that has frequently been observed with other meiotic genes. For example, the meiotic recombinase DMC1 conserved from yeasts to humans is absent from the C. elegans, Drosophila melanogaster, and Sordaria macrospora genomes.

\section{Structure of MEIOB}

MEIOB possesses three OB-folds homologous to those of RPA1 (Souquet et al. 2013; Luo et al. 2013). Similar to RPA1, MEIOB possesses two OB-folds and a long Cterminal OB-fold with a 30 -amino acid insertion containing a putative zinc ion-binding domain (see Fig. 2). We identified this motif in the human MEIOB sequence using the Sequence Similarity DataBase of the Kyoto Encyclopedia of Genes and Genomes (KEGG SSDB, http://www.kegg.jp/kegg/ssdb). This C-2X-C-10X-C-2X-C motif resembles that of a double zinc ribbon (DZR, unpublished data) and differs from that present in RPA1 only in the number of residues between the cysteines. We identified this motif in all metazoan MEIOB as well as in the MEIOB-like protein of the M. verticillata. The existence of this motif reinforces the hypothesis of a common origin between MEIOB and RPA1 even though MEIOBs and MEIOB-likes form a monophyletic group distinct from RPA1. The role of the putative zinc ion-binding domain of MEIOB should be the subject of future work to specify MEIOB functions during meiotic HR.

Compared with the very similar OB-fold domains, RPA1 and MEIOB significantly differ in the N-terminal parts of the proteins. The well-conserved N-terminal domain of RPA1 is absent in MEIOB. This domain ensures specific interactions between RPA1 and numerous partners such as DNA polymerase alpha and checkpoint proteins including ATRIP, RAD9 (53BP1), the MRE11/RAD50/NBS1 complex, and the tumor suppressor p53 (Kim et al. 1996; Bochkareva et al. 2002; Xu et al. 2003). This absence of such an N-terminal domain suggests that MEIOB developed its own specific interactions with a limited set of partners. This difference may support the existence of a distinct role for MEIOB and partially explain why RPA1 cannot compensate for the absence of MEIOB during meiosis in $\mathrm{Meiob}^{-/-}$mice (Souquet et al. 2013; Luo et al. 2013). The immunoprecipitation of MEIOB from testis extracts revealed an interaction between MEIOB and RPA2, a subunit of the RPA complex, and SPATA22, a meiosisspecific factor essential to prophase I progression (Luo et al. 2013; La Salle et al. 2012). However, the role and the nature of these interactions remain to be determined.

\section{Putative roles of MEIOB}

We identified Meiob as a meiosis-specific gene based on its differential expression between male and female embryonic germ cells at the time when only female germ cells have entered meiosis (Souquet et al. 2013). Luo et al. identified MEIOB through a systematical proteomic screen of meiosisspecific chromatin-associated proteins performed on mouse testes (Luo et al. 2013). The sterility of male and female $M e i o b^{-/-}$mice is due to zygotene/pachytene arrest in prophase I of meiosis. MEIOB is essential to the repair of meiotic DSBs 


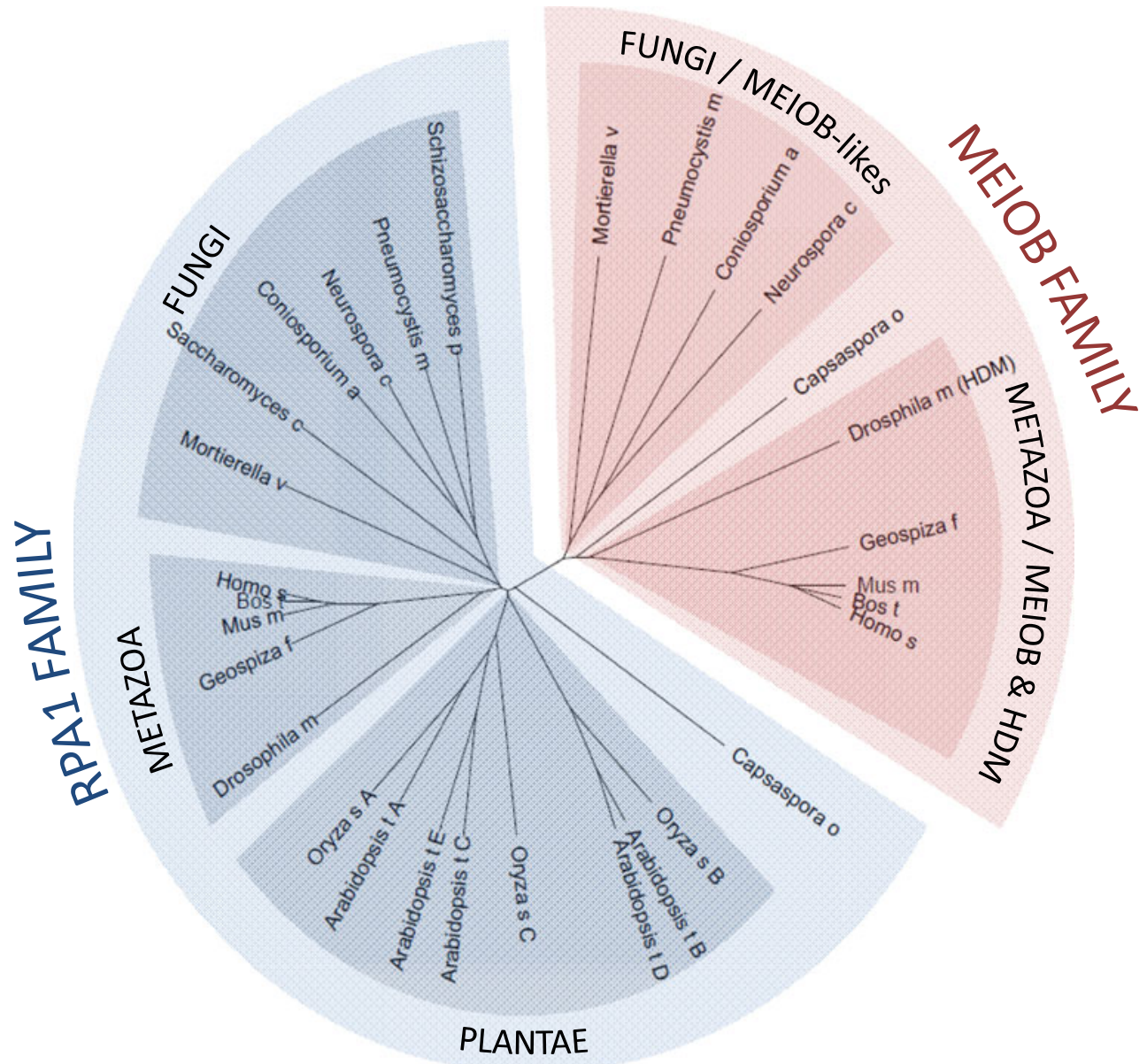

Fig. 3 Phylogenetic relationship between RPA and MEIOB homologs. Multiple alignments of full-length MEIOB and RPA1 protein were processed with Clustal Omega (http://www.ebi.ac.uk/Tools/msa/ clustalo/). The phylogenetic tree obtained with Clustal Omega was visualized with Archaeopteryx Version 0.9901 beta (Han and Zmasek 2009). MEIOB and RPA1 proteins form distinct families. Represented species: H. sapiens, Homo sapiens; B. taurus, Bos taurus; M. musculus, Mus musculus; G. fortis, Geospiza fortis; D. melanogaster, Drosophilae

and to proceed through meiosis. MEIOB is located on chromosome axes from leptotene to pachytene (Souquet et al. 2013; Luo et al. 2013). The fact that MEIOB is recruited to meiotic chromosomes in a $\mathrm{Dmcl}^{-/-}$mutant which is unable to undergo strand invasion of the homologous sequence suggests that MEIOB could play an early role on the ssDNA formed during DNA resection after DSB formation (Souquet et al. 2013). However, given that MEIOB persists until the pachytene stage, a later role in the processing of joint molecules formed during strand invasion is also possible (Souquet et al. 2013; Luo et al. 2013). Interestingly, we reported that in the absence of MEIOB, the recombinases DMC1 and RAD51 are loaded but not maintained on chromosome axes while RPA foci persist, suggesting that DSBs are not repaired (Souquet et al. 2013). This observation is reinforced by similar observations described in a rat mutant lacking functional SPATA22, a MEIOB partner (Ishishita et al. 2014). These melanogaster; C. owczarzaki, Capsaspora owczarzaki; N. crassa, Neurospora crassa; C. apollinis, Coniosporium apollinis; P. murina, Pneumocystis murina; M. verticillata, Mortierella verticillata; S. pombe, Schizosaccharomyces pombe; S. cerevisiae, Saccharomyces cerevisiae; O. sativa, Oryza sativa; A. thaliana, Arabidopsis thaliana. The amino acid sequences and accession numbers are available in supplementary material (Sup. 1)

results may suggest that the stabilization of the meiotic recombinases requires MEIOB and SPATA22. However, an alternative explanation would be that in absence of efficient DNA repair, the recombinases would be removed from recombination intermediates by helicase activity. The fact that RAD51 is loaded and not removed in a $\mathrm{Dmcl}^{-/}$mutant that is blocked prior to strand invasion, may suggest that recombination intermediates are not arrested at the same step in $\mathrm{Meiob}^{-/}$ and $\mathrm{Dmcl}^{-/-}$mutants (Pittman et al. 1998).

We found that only a fraction of MEIOB co-localizes with RPA2, particularly at early prophase I and, similarly to RPA, MEIOB is present on unsynapsed DNA (Souquet et al. 2013). These observations question whether MEIOB and RPA are loaded together on ssDNA formed on both sides of a DSB or individually loaded on each side of the DSB to provide different identities. We can also consider that similarly to AtRPA1c and AtRPA1a in A. thaliana, RPA1 and MEIOB 


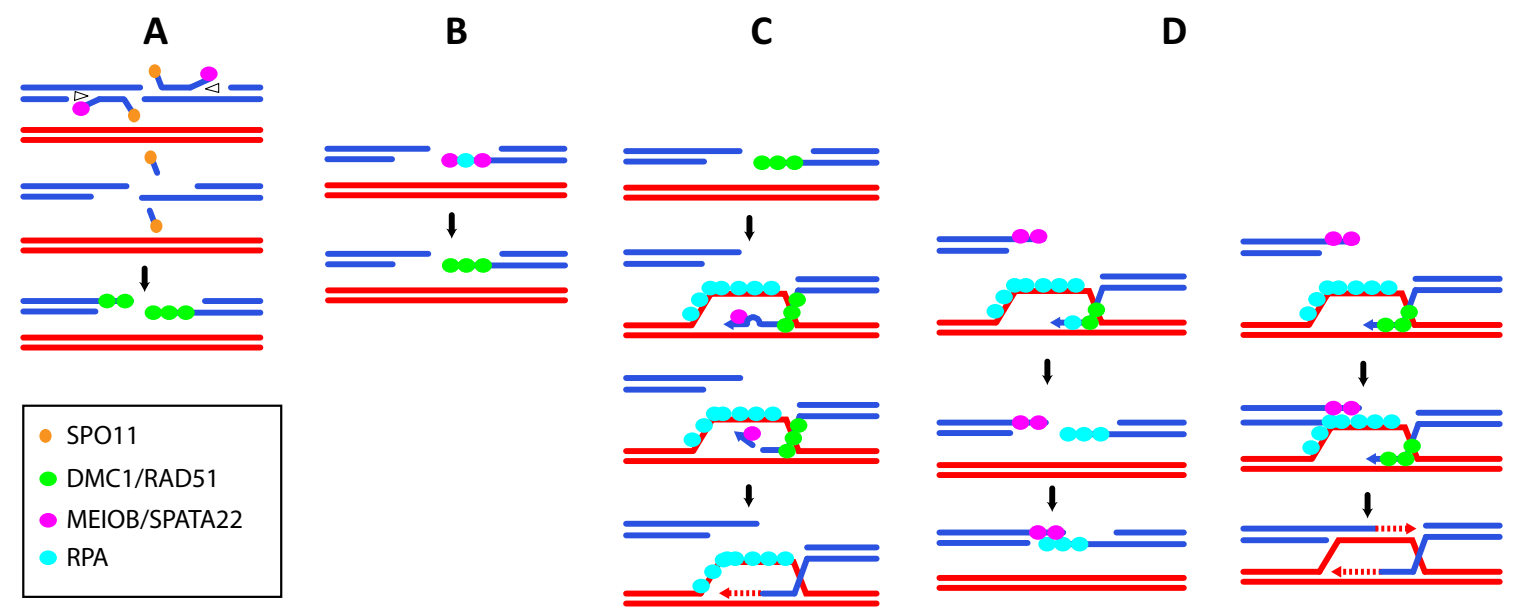

Fig. 4 Putative roles for MEIOB during meiotic recombination. a MEIOB is loaded during the early steps of resection to release Spo11oligo through its $3^{\prime}$-exonuclease activity with the help of a helicase (opened triangle). b MEIOB is loaded with or without RPA on the 3'ssDNA tail. c MEIOB 3'-exonuclease activity removes the $3^{\prime}$ end of the invading strand in the presence of mismatches formed between the donor and invading strand to allow initiation of DNA synthesis. $\mathbf{d}$ MEIOB is loaded on one side of the broken end to allow strand annealing or secondend capture

Nonetheless, 3 '-ssDNA tails need to be processed during meiotic homologous recombination. Luo et al. proposed that the putative exonuclease activity of MEIOB could process $3^{\prime}$ flaps formed after DNA synthesis during synthesis-dependent strand annealing (SDSA) or during $\mathrm{dHJ}$ formation. This activity may also be used after invasion of the homologous chromosomes, when sequence divergences between the invading strand and the donor induce the formation of DNA mismatches. Consequently the presence of these mismatches can induce the formation of a $3^{\prime}$ flap. In S. cerevisiae, it has been shown that during mitotic HR these $3^{\prime}$ flaps are processed by the endonuclease activity of the Rad1-Rad10 heterodimer to allow DNA synthesis to proceed and progress via recombination (Ivanov and Haber 1995). Through its nuclease activity, MEIOB may be involved in processing the $3^{\prime}$ flaps formed during strand invasion in meiosis (Fig. 4). Moreover, the MRE11 3'-exonuclease activity has been shown to be involved in the release of SPO11-oligonucleotides during meiotic DSB processing (Garcia et al. 2011). In a similar manner, MEIOB 3'-exonuclease activity with the help of a helicase could participate in early DSB processing (Fig. 4). Independently of its putative nuclease activity, Luo et al. proposed that due to its interaction with RPA2, MEIOB could provide a physical connection between an RPA-coated D-loop and the second broken end (Luo et al. 2013). An alternative possibility is that, similar to what has been proposed for AtRPA1a, MEIOB could be loaded on the second end and interact specifically with a RAD52-like factor and/or could possess an activity to anneal the second DSB end (Fig. 4). In addition, little is known about SPATA22. Similarly to MEIOB, SPATA22 is essential for meiotic HR and its invalidation induces a phenotype similar to that of MEIOB (La Salle et al. 2012; Ishishita et al. 2014). Moreover, the stabilities of 
MEIOB and SPATA22 are dependent on each other strongly suggesting that SPATA22 and MEIOB form a complex (Luo et al. 2013 and personal observations). MEIOB and SPATA22 show only a partial co-localization with RPA and are essential to meiotic HR. This observation suggests that MEIOB/ SPATA22 and the RPA complex can act both independently and together on DNA. Deciphering the role of SPATA22 during meiosis will be essential to better understand the function of the MEIOB/SPATA22 complex during meiotic homologous recombination.

\section{Conclusion}

In this review, we describe RPA-like proteins from different species. Studies in plants support the hypothesis that multiplying RPA family members may provide more specificity to the regulation of DNA metabolism and yield a larger panel of interacting factors. The existence of RPA4 in mammalian cells and MEIOB in metazoans and many fungi strongly suggests that this multiplicity is not restricted to plants. Because meiotic recombination is a conserved mechanism among species, we can investigate whether the role of MEIOB in species devoid of MEIOB could be supported by RPA. Conversely, MEIOB could also provide species-specific features to meiotic recombination. As an example, S. cerevisiae does not possess MEIOB. Interestingly, among the large panel of generated scRPA mutants and that affect specific functions, no meiosis-specific mutant has been identified (Soustelle et al. 2002). Based on the existence of homologies between scRPA and MEIOB which are absent in mammalian RPA, we also sought to identify specific meiotic domains in scRPA. Unfortunately, to date, the mutations of the putative domain tested did not allow us to identify any residues with a specific meiotic function in scRPA. These data would support the hypothesis that the presence of MEIOB provides species-specific features to the meiotic process, a hypothesis that will require further investigations. To better understand the role of MEIOB, it would be informative to know whether its recruitment is restricted to meiotic DSBs. Studying MEIOB localization during the repair of different types of DNA damage induced in mammalian mitotic cells after introducing $M E I O B$ by transfection or in meiotic cells lacking meiotic DSBs (i.e., Spo11-/mice) should shed light on the minimal requirements for in vivo MEIOB recruitment. Numerous uncertainties persist regarding the existence and putative purposes of different ssDNA-binding factors between mitotic and meiotic HR. Although RPA and MEIOB can partly co-localize on the meiotic chromosome axis, details of the dynamics of the various factors involved in ssDNA metabolism during meiosis are unclear. In this regard, deciphering whether MEIOB or RPA1 arrives and binds ssDNA first would be highly informative.

Understanding the nature and the role of specific ssDNAinteracting factors will provide essential key elements to improve our knowledge of the dynamics and regulation of meiotic recombination. This will also afford an original approach for deepening our understanding of the specific roles of canonical RPAs.

Acknowledgments We thank Matt Neale for providing critical comments regarding the manuscript. We thank members of Livera's lab for the technical support provided and acknowledge the funding provided by la Ligue Regionale Contre le Cancer. J.R. and E.A. are funded by the IRTELIS fellowships (CEA), and E.A. is funded by Fondation ARC.

\section{Compliance with ethical standards}

Conflict of interest The authors declare that they have no competing interests.

Ethics approval and consent to participate All applicable international, national, and /or institutional guidelines for the care and use of animals were followed.

This article does not contain any studies with human participants performed by any of the authors.

Open Access This article is distributed under the terms of the Creative Commons Attribution 4.0 International License (http:// creativecommons.org/licenses/by/4.0/), which permits unrestricted use, distribution, and reproduction in any medium, provided you give appropriate credit to the original author(s) and the source, provide a link to the Creative Commons license, and indicate if changes were made.

\section{References}

Aklilu BB, Soderquist RS, Culligan KM (2014) Genetic analysis of the replication protein A large subunit family in Arabidopsis reveals unique and overlapping roles in DNA repair, meiosis and DNA replication. Nucleic Acids Res 42:3104-3118

Alani E, Thresher R, Griffith JD, Kolodner RD (1992) Characterization of DNA-binding and strand-exchange stimulation properties of $y$ RPA, a yeast single-strand-DNA-binding protein. J Mol Biol 227: $54-71$

Anderson LK, Reeves A, Webb LM, Ashley T (1999) Distribution of crossing over on mouse synaptonemal complexes using immunofluorescent localization of MLH1 protein. Genetics 151:1569-1579

Bartrand AJ, Iyasu D, Marinco SM, Brush GS (2006) Evidence of meiotic crossover control in Saccharomyces cerevisiae through Mec1mediated phosphorylation of replication protein A. Genetics 172: 27-39

Bishop DK, Park D, Xu L, Kleckner N (1992) DMC1: a meiosis-specific yeast homolog of $\mathrm{E}$. coli recA required for recombination, synaptonemal complex formation, and cell cycle progression. Cell 69:439-456

Bochkarev A, Bochkareva E, Frappier L, Edwards AM (1999) The crystal structure of the complex of replication protein A subunits RPA32 and RPA14 reveals a mechanism for single-stranded DNA binding. EMBO J 18:4498-4504

Bochkareva E, Korolev S, Lees-Miller SP, Bochkarev A (2002) Structure of the RPA trimerization core and its role in the multistep DNAbinding mechanism of RPA. EMBO J 21:1855-1863

Borde V (2007) The multiple roles of the Mre11 complex for meiotic recombination. Chromosome Res 15:551-563 
Brown MS, Bishop DK (2014) DNA strand exchange and reca homologs in meiosis. Cold Spring Harb Perspect Biol 7:a016659. doi:10.1101/ cshperspect.a016659

Carballo JA, Panizza S, Serrentino ME, Johnson AL, Geymonat M, Borde V, Klein F, Cha RS (2013) Budding yeast ATM/ATR control meiotic double-strand break (DSB) levels by down-regulating Rec114, an essential component of the DSB-machinery. PLoS Genet 9:e1003545

Chang Y, Gong L, Yuan W, Li X, Chen G, Li X, Zhang Q, Wu C (2009) Replication protein A (RPA1a) is required for meiotic and somatic DNA repair but is dispensable for DNA replication and homologous recombination in rice. Plant Physiol 151:2162-2173

Cloud V, Chan YL, Grubb J, Budke B, Bishop DK (2012) Rad51 is an accessory factor for Dmc1-mediated joint molecule formation during meiosis. Science 337:1222-1225

Cole F, Baudat F, Grey C, Keeney S, de Massy B, Jasin M (2014) Mouse tetrad analysis provides insights into recombination mechanisms and hotspot evolutionary dynamics. Nat Genet 46:1072-1080

Cole F, Kauppi L, Lange J, Roig I, Wang R, Keeney S, Jasin M (2012) Homeostatic control of recombination is implemented progressively in mouse meiosis. Nat Cell Biol 14:424-430

Da Ines O, Degroote F, Goubely C, Amiard S, Gallego ME, White CI (2013) Meiotic recombination in arabidopsis is catalysed by DMC1, with RAD51 playing a supporting role. PLoS Genet 9:e1003787

De Muyt A, Zhang L, Piolot T, Kleckner N, Espagne E, Zickler D (2014) E3 ligase Hei10: a multifaceted structure-based signaling molecule with roles within and beyond meiosis. Genes Dev 28:1111-1123

Dong J, Park JS, Lee SH (1999) In vitro analysis of the zinc-finger motif in human replication protein A. Biochem J 337:311-317

Eggler AL, Inman RB, Cox MM (2002) The Rad51-dependent pairing of long DNA substrates is stabilized by replication protein A. J Biol Chem 277:39280-39288

Erdile LF, Heyer WD, Kolodner R, Kelly TJ (1991) Characterization of a cDNA encoding the 70-kDa single-stranded DNA-binding subunit of human replication protein A and the role of the protein in DNA replication. J Biol Chem 266:12090-12098

Eschbach V, Kobbe D (2014) Different replication protein A complexes of Arabidopsis thaliana have different DNA-binding properties as a function of heterotrimer composition. Plant Cell Physiol 55:1460 1472

Evans E, Alani E (2000) Roles for mismatch repair factors in regulating genetic recombination. Mol Cell Biol 20:7839-7844

Fan J, Pavletich NP (2012) Structure and conformational change of a replication protein A heterotrimer bound to ssDNA. Genes Dev 26:2337-2347

Gaines WA, Godin SK, Kabbinavar FF, Rao T, VanDemark AP, Sung P, Bernstein KA (2015) Promotion of presynaptic filament assembly by the ensemble of S. cerevisiae Rad51 paralogues with Rad52. Nat Commun 6:7834. doi: $10.1038 /$ ncomms 8834

Garcia V, Gray S, Allison RM, Cooper TJ, Neale MJ (2015) Tel1mediated interference suppresses clustered meiotic double-strandbreak formation. Nature 520:114-118. doi:10.1038/nature13993

Garcia V, Phelps SE, Gray S, Neale MJ (2011) Bidirectional resection of DNA double-strand breaks by Mre11 and Exo1. Nature 479:241244. doi:10.1038/nature10515

Gasior SL, Olivares H, Ear U, Hari DM, Weichselbaum R, Bishop DK (2001) Assembly of RecA-like recombinases: distinct roles for mediator proteins in mitosis and meiosis. Proc Natl Acad Sci U S A 98: 8411-8418

Gasior SL, Wong AK, Kora Y, Shinohara A, Bishop DK (1998) Rad52 associates with RPA and functions with rad55 and rad57 to assemble meiotic recombination complexes. Genes Dev 12:2208-2221

Golub EI, Gupta RC, Haaf T, Wold MS, Radding CM (1998) Interaction of human rad51 recombination protein with single-stranded DNA binding protein, RPA. Nucleic Acids Res 26:5388-5393
Gopalakrishnan AM, Kumar N (2013) Opposing roles for two molecular forms of replication protein A in Rad51-Rad54-mediated DNA recombination in Plasmodium falciparum. MBio 4:e00252-13

Guo S, Zhang Y, Yuan F, Gao Y, Gu L, Wong I, Li GM (2006) Regulation of replication protein A functions in DNA mismatch repair by phosphorylation. J Biol Chem 281:21607-21616

Haber J (2014) RPA and Rad51 assembly can be assayed IN VIVO. In: Scholl S (ed) Genome stability. Garland Science, New York, pp 5860

Han MV, Zmasek CM (2009) phyloXML: XML for evolutionary biology and comparative genomics. BMC Bioinformatics 10:356

Haring SJ, Humphreys TD, Wold MS (2010) A naturally occurring human RPA subunit homolog does not support DNA replication or cell-cycle progression. Nucleic Acids Res 38:846-858

Henderson KA, Kee K, Maleki S, Santini PA, Keeney S (2006) Cyclindependent kinase directly regulates initiation of meiotic recombination. Cell 125:1321-1332

Heyer WD, Kolodner RD (1989) Purification and characterization of a protein from Saccharomyces cerevisiae that binds tightly to singlestranded DNA and stimulates a cognate strand exchange protein. Biochemistry 28:2856-2862

Heyer WD, Rao MR, Erdile LF, Kelly TJ, Kolodner RD (1990) An essential Saccharomyces cerevisiae single-stranded DNA binding protein is homologous to the large subunit of human RP-A. EMBO J 9:2321-2329

Holloway JK, Sun X, Yokoo R, Villeneuve AM, Cohen PE (2014) Mammalian CNTD1 is critical for meiotic crossover maturation and deselection of excess precrossover sites. J Cell Biol 205:633641

Ishibashi T, Kimura S, Sakaguchi K (2006) A higher plant has three different types of RPA heterotrimeric complex. J Biochem 139: 99-104

Ishishita S, Matsuda Y, Kitada K (2014) Genetic evidence suggests that Spata22 is required for the maintenance of Rad51 foci in mammalian meiosis. Sci Rep 4:6148

Ivanov EL, Sugawara N, White CI, Fabre F, Haber JE (1994) Mutations in XRS2 and RAD50 delay but do not prevent mating-type switching in Saccharomyces cerevisiae. Mol Cell Biol 14:34143425

Ivanov EL, Haber JE (1995) RAD1 and RAD10, but not other excision repair genes, are required for double-strand break-induced recombination in Saccharomyces cerevisiae. Mol Cell Biol 15:2245-2251

Jensen RB, Carreira A, Kowalczykowski SC (2010) Purified human BRCA2 stimulates RAD51-mediated recombination. Nature 467: $678-683$

Joyce EF, Tanneti SN, McKim KS (2009) Drosophila hold'em is required for a subset of meiotic crossovers and interacts with the dna repair endonuclease complex subunits MEI-9 and ERCC1. Genetics 181: 335-340

Kallberg M, Wang H, Wang S, Peng J, Wang Z, Lu H, Xu J (2012) Template-based protein structure modeling using the RaptorX web server. Nat Protoc 7:1511-1522

Kauppi L, Jasin M, Keeney S (2013) How much is enough? Control of DNA double-strand break numbers in mouse meiosis. Cell Cycle 12:2719-2720

Keshav KF, Chen C, Dutta A (1995) Rpa4, a homolog of the 34kilodalton subunit of the replication protein A complex. Mol Cell Biol 15:3119-3128

Kim DK, Stigger E, Lee SH (1996) Role of the 70-kDa subunit of human replication protein A (I). Single-stranded DNA-binding activity, but not polymerase stimulatory activity, is required for DNA replication. J Biol Chem 271:15124-15129

Kurzbauer MT, Uanschou C, Chen D, Schlogelhofer P (2012) The recombinases DMC1 and RAD51 are functionally and spatially separated during meiosis in Arabidopsis. Plant Cell 24:2058-2070 
La Salle S, Palmer K, O’Brien M, Schimenti JC, Eppig J, Handel MA (2012) Spata22, a novel vertebrate-specific gene, is required for meiotic progress in mouse germ cells. Biol Reprod 86:45. doi:10. 1095/biolreprod

Lange J, Pan J, Cole F, Thelen MP, Jasin M, Keeney S (2011) ATM controls meiotic double-strand-break formation. Nature 479:237240

Lao JP, Oh SD, Shinohara M, Shinohara A, Hunter N (2008) Rad52 promotes postinvasion steps of meiotic double-strand-break repair. Mol Cell 29:517-524

Luo M, Yang F, Leu NA, Landaiche J, Handel MA, Benavente R, La Salle S, Wang PJ (2013) MEIOB exhibits single-stranded DNAbinding and exonuclease activities and is essential for meiotic recombination. Nat Commun 4:2788

Martini E, Diaz RL, Hunter N, Keeney S (2006) Crossover homeostasis in yeast meiosis. Cell 126:285-295

Mehta A, Haber JE (2014) Sources of DNA double-strand breaks and models of recombinational DNA repair. Cold Spring Harb Perspect Biol 6:a016428

Moens PB, Kolas NK, Tarsounas M, Marcon E, Cohen PE, Spyropoulos B (2002) The time course and chromosomal localization of recombination-related proteins at meiosis in the mouse are compatible with models that can resolve the early DNA-DNA interactions without reciprocal recombination. J Cell Sci 115:1611-1622

Murakami H, Keeney S (2014) Temporospatial Coordination of Meiotic DNA Replication and Recombination via DDK Recruitment to Replisomes. Cell 158:861-873

Oliver-Bonet M, Campillo M, Turek PJ, Ko E, Martin RH (2007) Analysis of replication protein A (RPA) in human spermatogenesis. Mol Hum Reprod 13:837-844

Osman K, Sanchez-Moran E, Mann SC, Jones GH, Franklin FC (2009) Replication protein A (AtRPA1a) is required for class I crossover formation but is dispensable for meiotic DNA break repair. EMBO J 28:394-404

Page SL, Hawley RS (2003) Chromosome choreography: the meiotic ballet. Science 301:785-789

Petalcorin MI, Sandall J, Wigley DB, Boulton SJ (2006) CeBRC-2 stimulates D-loop formation by RAD-51 and promotes DNA singlestrand annealing. J Mol Biol 361:231-242

Philipova D, Mullen JR, Maniar HS, Lu J, Gu C, Brill SJ (1996) A hierarchy of SSB protomers in replication protein A. Genes Dev 10:2222-2233

Pittman DL, Cobb J, Schimenti KJ, Wilson LA, Cooper DM, Brignull E, Handel MA, Schimenti JC (1998) Meiotic prophase arrest with failure of chromosome synapsis in mice deficient for Dmc1, a germlinespecific RecA homolog. Mol Cell 1:697-705

Plug AW, Peters AH, Keegan KS, Hoekstra MF, de Boer P, Ashley T (1998) Changes in protein composition of meiotic nodules during mammalian meiosis. J Cell Sci 111:413-423

Qiao H, Prasada Rao HB, Yang Y, Fong JH, Cloutier JM, Deacon DC, Nagel KE, Swartz RK, Strong E, Holloway JK, Cohen PE, Schimenti J, Ward J, Hunter N (2014) Antagonistic roles of ubiquitin ligase HEI10 and SUMO ligase RNF212 regulate meiotic recombination. Nat Genet 46:194-199

Rider SDJ, Cai X, Sullivan WJJ, Smith AT, Radke J, White M, Zhu G (2005) The protozoan parasite Cryptosporidium parvum possesses two functionally and evolutionarily divergent replication protein A large subunits. J Biol Chem 280:31460-31469

Rijkers T, Van Den Ouweland J, Morolli B, Rolink AG, Baarends WM, Van Sloun PP, Lohman PH, Pastink A (1998) Targeted inactivation of mouse RAD52 reduces homologous recombination but not resistance to ionizing radiation. Mol Cell Biol 18:6423-6429

Salas TR, Petruseva I, Lavrik O, Saintome C (2009) Evidence for direct contact between the RPA3 subunit of the human replication protein A and single-stranded DNA. Nucleic Acids Res 37:38-46
Schwacha A, Kleckner N (1994) Identification of joint molecules that form frequently between homologs but rarely between sister chromatids during yeast meiosis. Cell 76:51-63

Seeliger K, Dukowic-Schulze S, Wurz-Wildersinn R, Pacher M, Puchta $\mathrm{H}$ (2012) BRCA2 is a mediator of RAD51- and DMC1-facilitated homologous recombination in Arabidopsis thaliana. New Phytol 193:364-375

Sharan SK, Pyle A, Coppola V, Babus J, Swaminathan S, Benedict J, Swing D, Martin BK, Tessarollo L, Evans JP, Flaws JA, Handel MA (2004) BRCA2 deficiency in mice leads to meiotic impairment and infertility. Development 131:131-142

Shultz RW, Tatineni VM, Hanley-Bowdoin L, Thompson WF (2007) Genome-wide analysis of the core DNA replication machinery in the higher plants Arabidopsis and rice. Plant Physiol 144:16971714

Siaud N, Dray E, Gy I, Gerard E, Takvorian N, Doutriaux MP (2004) Brca2 is involved in meiosis in Arabidopsis thaliana as suggested by its interaction with Dmc1. EMBO J 23:1392-1401

Sneeden JL, Grossi SM, Tappin I, Hurwitz J, Heyer WD (2013) Reconstitution of recombination-associated DNA synthesis with human proteins. Nucleic Acids Res 41:4913-4925

Souquet B, Abby E, Herve R, Finsterbusch F, Tourpin S, Le Bouffant R, Duquenne C, Messiaen S, Martini E, Bernardino-Sgherri J, Toth A, Habert R, Livera G (2013) MEIOB targets single-strand DNA and is necessary for meiotic recombination. PLoS Genet 9:e1003784

Soustelle C, Vedel M, Kolodner R, Nicolas A (2002) Replication protein $\mathrm{A}$ is required for meiotic recombination in Saccharomyces cerevisiae. Genetics 161:535-547

Sugiyama T, Kantake N, Wu Y, Kowalczykowski SC (2006) Rad52mediated DNA annealing after Rad51-mediated DNA strand exchange promotes second ssDNA capture. EMBO J 25:5539-5548

Sugiyama T, New JH, Kowalczykowski SC (1998) DNA annealing by RAD52 protein is stimulated by specific interaction with the complex of replication protein A and single-stranded DNA. Proc Natl Acad Sci U S A 95:6049-6054

Sugiyama T, Zaitseva EM, Kowalczykowski SC (1997) A singlestranded DNA-binding protein is needed for efficient presynaptic complex formation by the Saccharomyces cerevisiae Rad51 protein. J Biol Chem 272:7940-7945

Sung P (1994) Catalysis of ATP-dependent homologous DNA pairing and strand exchange by yeast RAD51 protein. Science 265:1241-1243

Sung P (1997) Function of yeast Rad52 protein as a mediator between replication protein A and the Rad51 recombinase. J Biol Chem 272: 28194-28197

Suwaki N, Klare K, Tarsounas M (2011) RAD51 paralogs: roles in DNA damage signalling, recombinational repair and tumorigenesis. Semin Cell Dev Biol 22:898-905

Tarsounas M, Davies D, West SC (2003) BRCA2-dependent and independent formation of RAD51 nuclear foci. Oncogene 22:1115-1123

Thorslund T, West SC (2007) BRCA2: a universal recombinase regulator. Oncogene 26:7720-7730

Tsubouchi H, Ogawa H (1998) A novel mre11 mutation impairs processing of double-strand breaks of DNA during both mitosis and meiosis. Mol Cell Biol 18:260-268

Walther AP, Gomes XV, Lao Y, Lee CG, Wold MS (1999) Replication protein A interactions with DNA. 1. Functions of the DNA-binding and zinc-finger domains of the $70-\mathrm{kDa}$ subunit. Biochemistry 38 : 3963-3973

Wang X, Haber JE (2004) Role of Saccharomyces single-stranded DNAbinding protein RPA in the strand invasion step of double-strand break repair. PLoS Biol 2:E21

Weiner BM, Kleckner N (1994) Chromosome pairing via multiple interstitial interactions before and during meiosis in yeast. Cell 77:977-991

Wold MS (1997) Replication protein A: a heterotrimeric, single-stranded DNA-binding protein required for eukaryotic DNA metabolism. Annu Rev Biochem 66:61-92 
Wold MS, Kelly T (1988) Purification and characterization of replication protein $\mathrm{A}$, a cellular protein required for in vitro replication of simian virus 40 DNA. Proc Natl Acad Sci U S A 85:2523-2527

Xu X, Aprelikova O, Moens P, Deng CX, Furth PA (2003) Impaired meiotic DNA-damage repair and lack of crossing-over during spermatogenesis in BRCA1 full-length isoform deficient mice. Development 130:2001-2012
Yoshida K, Kondoh G, Matsuda Y, Habu T, Nishimune Y, Morita $\mathrm{T}$ (1998) The mouse RecA-like gene Dmcl is required for homologous chromosome synapsis during meiosis. Mol Cell $1: 707-718$

Youds JL, Boulton SJ (2011) The choice in meiosis-defining the factors that influence crossover or non-crossover formation. J Cell Sci 124: $501-513$ 